\title{
Numerical study of the phase diagram of a mixture of spherical and rodlike colloids
}

\author{
Peter Bolhuis and Daan Frenkel \\ FOM Institute for Atomic and Molecular Physics, Kruislaan 407, 1098 SJ Amsterdam, The Netherlands
}

(Received 27 May 1994; accepted 22 August 1994)

\begin{abstract}
Fluid-fluid phase separation in a simple model for a colloid dispersed in a solution of stiff rodlike polymers is studied by means of Gibbs ensemble Monte Carlo simulations. The fluid-solid transition for this model is computed using first-order perturbation theory. The phase diagrams clearly show that a stable critical point is not possible for polymer lengths smaller than $30 \%$ of the colloidal particle diameter. At very large rod length, the fluid coexistence curve can be derived directly from the hard-sphere equation of state. (C) 1994 American Institute of Physics.
\end{abstract}

\section{INTRODUCTION}

When a suspension of uncharged colloidal particles is mixed with a sufficiently concentrated solution of nonadsorbing polymer, a phase separation occurs, resulting in a dense colloidal phase and a phase that contains mainly polymer. ${ }^{1-3}$ This transition takes place because the increase in free energy associated with the condensation of the colloidal particles is more than offset by the decrease in free energy due to the gain of free volume accessible to the polymers.

A simple, yet qualitatively correct model of polymercolloid mixtures was first proposed by Asakura and Oosawa ${ }^{1}$ and subsequently analyzed by $\mathrm{Vrij}^{2}$ and Lekkerkerker et $a l^{3}$ In this model, the colloids are represented by hard spheres of radius $R_{c}=\sigma / 2$, while the polymers are assumed to be spheres of radius $R_{p}$ that are mutually interpenetrable, yet cannot penetrate the colloidal particles. As a consequence, every colloidal particle excludes a sphere of volume $\frac{4}{3} \pi\left(R_{c}+R_{p}\right)^{3}$ to the polymers. When two colloidal particles are brought together, these spherical volumes will overlap and the volume accessible to the polymer will increase. It is this increase in free volume that causes an effective attraction and the eventual phase separation of the colloids. Recent computer simulations of polymer-colloid mixtures ${ }^{4}$ indicate that the Asakura-Oosawa model is quite realistic if $R_{p}<R_{c}$.

Of course; the above mechanism for polymer-colloid phase separation is more general: spherical colloids and globular polymers are just the simplest example. In the present paper, we focus on another limiting example, namely a mixture of spherical colloids and thin rodlike polymers. In the limit that the polymers are infinitely thin, this mixture is expected to resemble the Asakura-Oosawa model, because the polymer-polymer excluded volume vanishes, while the polymer colloid excluded volume is a spherocylinder with a length to width ratio $1+L / \sigma$, where $L$ is the length of the rodlike polymers, and $\sigma$ the diameter of the colloidal spheres. As in the case of the flexible polymer-colloid mixture the free volume for the rodlike polymer can be increased by clustering of the colloids. One would therefore expect to see phase separation in such mixtures. However, although a light scattering study of a mixture of colloids and fairly rigid rodlike polymers was recently reported by Tracy et al., phase separation was not observed.
In the present paper we report a systematic numerical study of mixtures of hard-sphere colloids with thin rodlike polymers of varying length. The aim of the paper is threefold: first of all, we wish to establish if there is indeed a fluid-fluid phase separation in this mixture. This is not a priori obvious because hoth the spheres and the rodlike polymers are convex bodies. The existence of a fluid-fluid phase separation in the simplest mixture of convex bodies, viz. hard spheres, continues to be a matter of speculation. ${ }^{6,7}$ For other mixtures of convex bodies, firm data is lacking. A second aim of the paper is to provide a guide for experiments: we intend to indicate under what conditions (colloid density, polymer fugacity, $L / \sigma$ ratio) phase transitions are to be expected. We shall consider both the possibility of fluidfluid and fluid-solid transitions. Finally, we wish to verify if the theoretical description of polymer-colloid mixtures discussed in Ref. 3 can be generalized to rodlike polymers.

In our numerical study of possible fluid-fluid coexistence, we have made use of the Gibbs ensemble method of Panagiotopolous. ${ }^{8,9}$ However, as will be explained below, a naive implementation of this scheme is extremely inefficient in the present case. We have therefore generalized the Gibbs ensemble method to perform multiparticle exchanges, where the configurational bias Monte Carlo method ${ }^{10}$ is used to achieve a high acceptance probability of trial moves. This simulation scheme is described in the Appendix.

In Sec. II A we present the simulations of fluid-fluid demixing. The solid-liquid coexistence is discussed in Sec. II B. The application of first-order perturbation theory to the mixture is described in Sec. III A, followed by a discussion of the limiting case of infinitely long rods, $L \rightarrow \infty$. In Sec. III $\mathrm{C}$ we compare the simulation results with the theory.

\section{SIMULATIONS}

\section{A. Fluid-fluid coexistence}

In the model studied in our simulations the colloidal particles are represented by hard spheres with a diameter $\sigma$ while the rodlike particles are modeled by infinitely thin needles of length $L$. As these needles are infinitely thin, their mutual excluded volume vanishes. However, the needles cannot intersect with the hard core colloidal particles.

In order to estäblish fluid-fluid phase separation we performed modified Gibbs ensemble Monte Cärlo simulations 


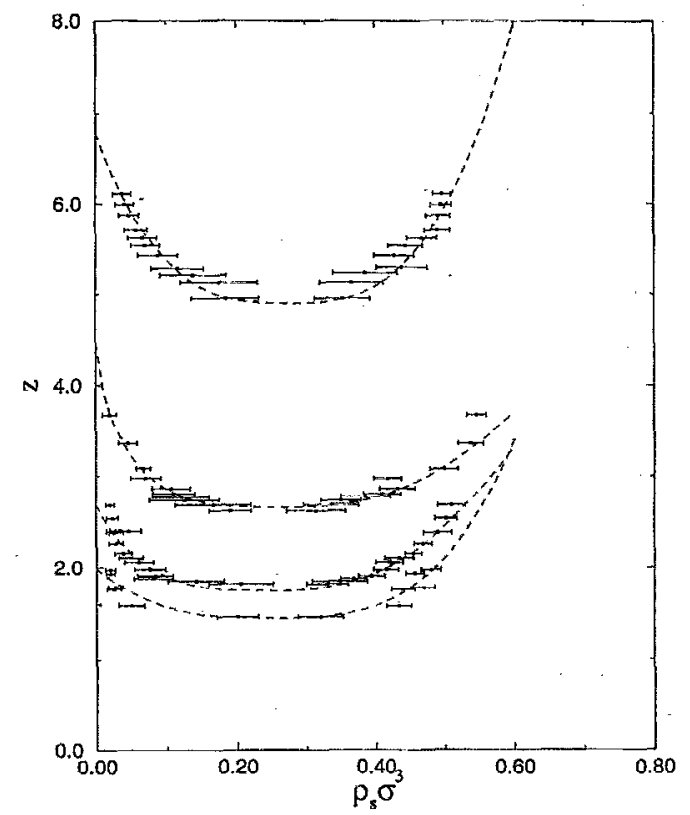

FIG. 1. Coexistence curves for mixtures of hard sphere and rods. On the $x$ axis the number density of hard spheres is set out. The fugacity of the rods $z$ is plotted vs the reduced number density of hard spheres, $\rho_{s} \sigma^{3}$. The data points refer to the Gibbs ensemble results. From top to bottom the sets of points denote the binodals for $L / \sigma=2,3,4$, and 5 . The dashed curves are fits to $\mathrm{Eq}$. (1) with an exponent $1 / 3$.

for rod lengths $L / \sigma=2,3,4,5,6$ at different fugacities (see the Appendix). The overall starting density of colloidal particles in the two boxes was $\rho \sigma^{3}=0.25$ which located the system in a two phase region of the phase diagram. The total number of spheres in the system was 110 . We did not fix the number of rod-like polymers, but rather kept their fugacity $z$ fixed. During the simulations the number of rods varied from 100 to 1000.

As a test, we measured the chemical potential of the needles and the spheres using Widoms particle insertion technique ${ }^{11}$ during a simulation. The chemical potential $\mu$ of the rods is directly related to the fugacity by $z=\exp \beta \mu$. We checked that the actual chemical potentials of the two species remained constant in both phases.

For equilibration of every system $10000 \mathrm{MC}$ cycles were executed, whereas 20000 cycles were performed for data collection. During a cycle every particle on average was moved once. In addition the average cycle contained 80 attempted particle exchanges and 1 volume exchange between the two phases. The maximum step sizes of the particle moves were adjusted in order to keep the acceptance probability around $30 \%-40 \%$. The acceptance probability of hard-sphere exchanges which involved the configurational bias Monte Carlo step was $\sim 5 \%$. This means that on average during every cycle $2 \%$ of the total number of particles was exchanged. The acceptance probability for the exchange of the needles was $\sim 40 \%$.

Figure I shows the fluid-fluid coexistence curves obtained in the Gibbs ensemble simulations. As the length of the needles is increased, the coexistence curves shift to lower fugacity. This is to be expected because the range of the depletion interaction increases with length. Therefore, fewer rods are needed to induce the phase separation.

Because of the analogy between this phase separation and the liquid-vapor coexistence in simple fluids we expect that the shape of the coexistence curve in the neighborhood of the critical point is given by the scaling form

$$
\rho_{l}-\rho_{g} \sim\left(z-z_{c}\right)^{\beta}
$$

where $\rho_{l}-\rho_{g}$ is the density difference between the dense and the dilute phase and we assumed $\beta=0.33$, the usual $3 D$ Ising critical exponent. From these simulations it is not possible to obtain a good estimate of the actual critical exponent. The dashed curves shown in Fig. 1 are fits of the scaling form (1).

The critical density shifts to lower values and the coexistence curves become more asymmetrical with increasing needle length. For very long rods every sphere will be expelled from the dilute vaporlike phase, which causes the critical density to go asymptotically to zero for $L \rightarrow \infty$. This limit will be discussed in more detail in Sec. III B.

\section{B. Solid-fluid transition}

Although the Gibbs ensemble simulations described above indicate that a fluid-fluid-like demixing occurs in the sphere-needle mixture, it is not obvious that this phase equilibrium can indeed be observed in experiments. In particular, the fluid-solid transition might take place before the fluidfluid two phase region is reached. It is therefore essential to study the stability of the fluid phase with respect to the solid. To locate the freezing transition we must compute the Helmholtz free energy of both the fluid and solid phases. This free energy is best computed by thermodynamic integration, using the hard-sphere system as a reference. The Helmholtz free energy of a sphere-needle mixture at volume fraction $\phi$ of spheres and fugacity $z$ of the needles is given by

$$
\begin{aligned}
F(\phi, z) & =F_{\mathrm{HS}}(\phi)+\int_{0}^{z} d z^{\prime} \frac{\partial F}{\partial z^{\prime}} \\
& =F_{\mathrm{HS}}(\phi)-\int_{0}^{z} d z^{\prime}\left\langle V_{\text {free }}\right\rangle,
\end{aligned}
$$

where $F_{\mathrm{HS}}$ is the hard-sphere reference energy and $\left\langle V_{\text {free }}\right\rangle$ the average free volume available to the rods. This free volume is a function of the fugacity. We performed simulations to obtain the free volume for a rod in the dense fluid and the solid phase at different fugacities. For the solid we assumed a face-centered-cubic ( $\mathrm{fcc}$ ) crystal structure which is presumably the most stable solid phase of hard spheres.

Figure 2 shows the measured average free volume fraction for one rod as a function of the hard-sphere volume fraction for several needle fugacities. The free volume available to the needles is almost independent of the fugacity: The fluid structure is therefore only slightly effected by the addition of needles. Because the free volume is effectively independent of fugacity we can use first-order perturbation theory to calculate the free energy at higher fugacities. Therefore Eq. (2) reduces to

$$
F(\phi, z)=F_{\mathrm{HS}}(\phi)-z\left\langle V_{\mathrm{free}}\right\rangle_{\mathrm{HS}},
$$




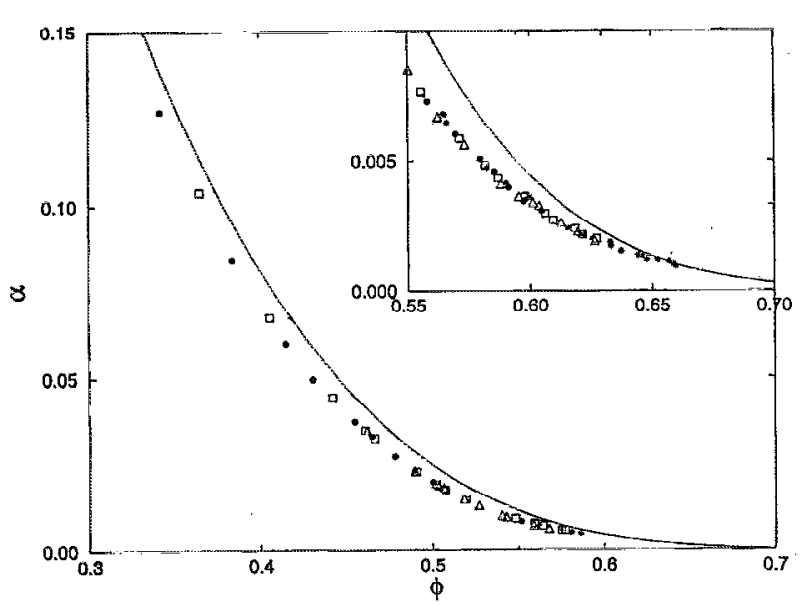

FIG. 2. Simulated free volume fraction $\alpha$ for infinitely thin rods of length $L / \sigma=2$ as a function of hard-sphere volume fraction $\phi$. Simulation parameters as given in the text. The main figure gives the free volume in the fluid phase, whereas in the inset the much smaller free volume of the solid phase is plotted. The symbols refer to different rod fugacity: $z=2.7 ; \square z=7.4$; $\Delta z=12.2$; and ${ }^{*} z=20.1$. The sulid curve is the scaled particle theory approximation to the free volume for $L / \sigma=2$. Note that the relative difference of the simulated data and the SPT curve is larger in the solid phase.

where $\left\langle V_{\text {free }}\right\rangle_{\text {Hs }}$ is the free volume for one rod in the hardsphere reference system. From this equation the coexistence densities of buth the fluid and the solid phases can be obtained by equating the pressure and chemical potentials in both phases. For the fluid hard-sphere equation of state we used the Carnahan-Starling expression ${ }^{12}$ whereas the solid hard-sphere equation of state and reference free energy were obtained from simulation data. ${ }^{13,14}$

Although $\left\langle V_{\text {free }}\right\rangle_{\text {HS }}$ could be obtained from simulation data, we found that in the fluid phase it could be approximated quite well by scaled particle theory (SPT). ${ }^{15}$ In the solid phase the SPT considerably overestimates the free volume. This is not surprising as the theory was derived for the fluid region. However, as the free volume in the solid is very small anyway, the overestimate will not alter the thermodynamic properties of the mixture unless one considers very high polymer fugacity. ${ }^{4}$

\section{THEORY}

\section{A. Perturbation theory}

To compute the fluid-fluid coexistence curves of the rod-sphere mixture we use the perturbation theory approach developed by Lekkerkerker et al. ${ }^{3,7}$ Consider a system consisting of two phases with volume $V^{1}$ and $V^{\text {II }}$ containing, respectively, $N_{r}^{\mathrm{I}}, N_{r}^{\mathrm{II}}$ rods of length $L$ and $N_{s}^{\mathrm{I}}, N_{s}^{\mathrm{II}}$ spheres with diameter $\sigma$. These phases are in osmotic equilibrium with a reservoir containing an ideal gas of rods at reduced pressure $\beta P^{0}$ which is equal to the fugacity $z=\exp (-\beta \mu)$, where $\mu$ is the chemical potential of the rods and $\beta=1 / k T$ is the inverse temperature.

In order to simplify the notation in the following equations: $N_{s}, N_{r}$, and $V$ for a single not yet specified phase will be used. As we are imposing the rod fugacity, the proper thermodynamic quantity is the grand canonical partition function

$$
\begin{aligned}
\Xi\left(N_{s}, \mu, V\right)= & \sum_{m=0}^{\infty} \frac{z^{m}}{m !} \int d r^{N_{r}} \\
& \times \exp \left(-\beta U_{\mathrm{HS}}\right) Z\left(r^{N_{s}}, m, V\right),
\end{aligned}
$$

where $U_{\mathrm{HS}}$ is the interaction energy between the hard spheres and $Z\left(r^{N}, m, V\right)$ is the configurational partition function of $m$ rods in a particular configuration of the hard spheres. Because the rods are ideal this partition function is the $m$ th power of the free volume available to one rod:

$$
Z\left(r^{N}, m, V\right)=\left[V_{\text {free }}\left(r^{N_{s}}\right)\right]^{m} .
$$

The grand canonical partition function becomes

$$
\Xi\left(N_{s}, \mu, V\right)=\int d r^{N_{r}} \exp \left(-\beta U_{\mathrm{HS}}\right) \exp \left[z V_{\mathrm{free}}\left(r^{N_{s}}\right)\right]
$$

Using a van der Waals type mean-field approximation which replaces $V_{\text {free }}\left(r^{N_{s}}\right)$ by its average value in the corresponding unperturbed hard-sphere reference system, we obtain the grand potential

$$
\begin{aligned}
\beta \Omega\left(N_{s}, \mu, V\right) & =-\ln \Xi\left(N_{s}, \mu, V\right) \\
& \approx-\ln \left\langle\exp \left(-\beta U_{\mathrm{HS}}\right)\right\rangle-z\left\langle V_{\mathrm{free}}\right\rangle \\
& =\beta F_{\mathrm{HS}}\left(N_{s}, V\right)-z \alpha V .
\end{aligned}
$$

Here the brackets stand for an ensemble average over the sphere configurations, $F_{\mathrm{HS}}$ is the free energy of the hard spheres, which only depends on the density $\rho_{s}=N_{s} / V$, and $\alpha$ is the average fraction of the total volume available for a rod. This recipe is equivalent to applying first-order perturbation theory.

To calculate the phase equilibria, one needs the pressure and the chemical potential of the spheres

$$
\begin{aligned}
& \beta P=-\beta\left(\frac{\partial \Omega}{\partial V}\right)_{N_{s}, \mu, T}=\beta P_{\mathrm{HS}}+z\left(\alpha-\rho_{s} \frac{d \alpha}{d \rho_{s}}\right), \\
& \beta \mu_{s}=\beta\left(\frac{\partial \Omega}{\partial N_{s}}\right)_{V, \mu, T}=\beta \mu_{\mathrm{HS}}-z \frac{d \alpha}{d \rho_{s}} .
\end{aligned}
$$

For the pressure of the hard-sphere reference system $P_{\text {HS }}$ we use the Carnahan-Starling equation of state

$$
\frac{\beta P_{\mathrm{HS}}}{\rho_{s}}=\frac{1+\phi+\phi^{2}-\phi^{3}}{(1-\phi)^{3}},
$$

where $\phi=\rho_{s} \frac{1}{6} \pi \sigma^{3}$ is the volume fraction of hard spheres. The chemical potential of the hard-sphere fluid can be obtained from the standard thermodynamic relations. For the free volume fraction $\alpha$ one can use the scaled particle theory for one rod in a solution of spheres: ${ }^{15}$

$$
\beta \mu^{\mathrm{ex}}=-\ln \alpha=-\ln (1-\phi)+\frac{3}{2} \frac{L}{\sigma} \frac{\phi}{1-\phi} .
$$

This is an underestimation of the chemical potential, therefore an overestimation of the free volume as we already saw 
in Fig. 2. Nevertheless, it turns out that this equation gives quite accurate results as we shall see in Sec. III C. Substitution of Eq. (11) in Eqs. (8) and (9) yields explicit expressions for the pressure and chemical potentials in terms of the volume fractions in phase I and phase II. The coexistence curves were obtained by equating the pressure and chemical potentials in both phases.

\section{B. The limit $L \rightarrow \infty$}

The liquid-vapor coexistence terminates in a critical point. If the rod length increases the critical density shifts to lower values. For $L \rightarrow \infty$ this density is proportional to $1 / L$, as can be derived from perturbation theory by setting the first and second derivative of the pressure [Eq. (8)] with respect to the density equal to zero. The critical volume fraction $\phi_{c}$ then follows as a function of $L$ :

$$
\phi_{c}=\frac{10+3 L / \sigma-3 \sqrt{4+12 L / \sigma+(L / \sigma)^{2}}}{16-12 L / \sigma} \stackrel{L \rightarrow \infty}{=} \frac{2 \sigma}{3 L} .
$$

Using this result we can obtain the critical rod fugacity from Eq. (8) as well:

$$
\begin{aligned}
z_{c} & =\frac{4 \sigma^{2}}{9 L^{2}} \frac{1+4 \phi_{c}+4 \phi_{c}^{2}-4 \phi_{c}^{3}+\phi_{c}^{4}}{1-\phi_{c}} \exp \left(\frac{3 L}{2 \sigma} \frac{\phi_{c}}{1-\phi_{c}}\right) \\
& =\frac{2 \sigma e}{3 L} .
\end{aligned}
$$

Another remarkable fact is that the theoretical coexistence curve has a simple asymptotic form for very long rod lengths. If the critical density becomes zero the hard-sphere vapor density also has to vanish. In this region the liquid vapor coexistence curve is casy to calculatc by equating the pressures of the liquid and the vapor phase. The chemical potential is not important in the limit of long rods because the coexistence density of the vapor is effectively independent of the chemical potential. In the vapor phase the pressure is simply equal to the fugacity of the rods because there are no spheres present. The pressure in the fluid phase follows from Eq. (8):

$$
\beta P=\beta P_{\mathrm{HS}}+z\left(\alpha-\phi \frac{d \alpha}{d \phi}\right) .
$$

In the $L \rightarrow \infty$ limit the free volume fraction $\alpha$ available to the rods in the liquid vanishes. The pressure of the fluid therefore reduces to the hard-sphere equation of state. Equating the pressure in both phases gives

$$
\beta P=\beta P_{\mathrm{HS}}=z \text {. }
$$

This result provides an alternative route to measure the hardsphere equation of state in experiments by measuring the rod concentration of the vapor phase as a function of the density of spheres in the liquid phase.

\section{Comparison with simulation results}

In Fig. 3 the binodal curves from first-order perturbation theory are compared with the Gibbs ensemble results. The critical points are well predicted for small lengths but be-

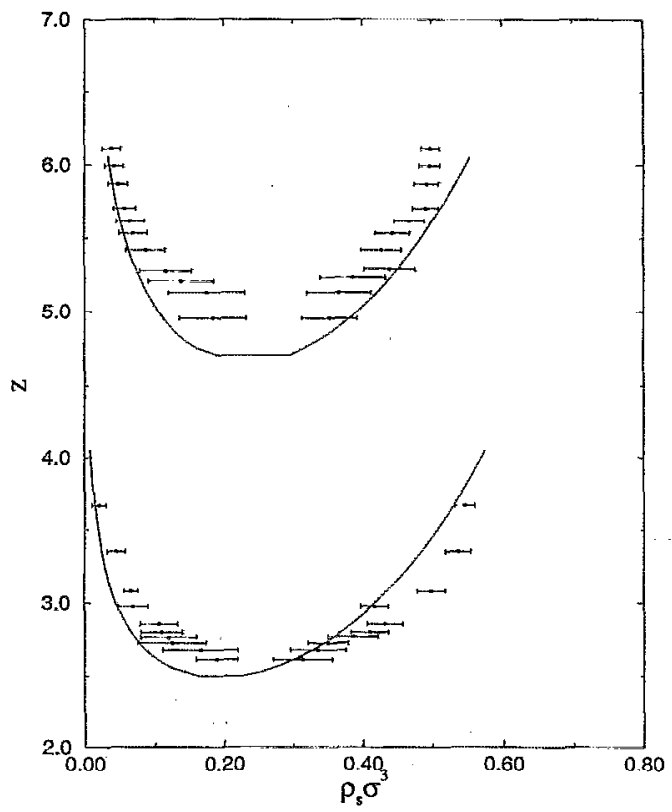

FIG. 3. Binodal curves obtained from perturbation theory compared with simulation results. The fugacity of the rods $z$ is plotted vs the reduced number density of hard spheres, $\rho_{s} \sigma^{3}$. The upper curve and points correspond to $L / \sigma=2$, the lower to $L / \sigma=3$.

come worse for longer rods. Because there is no adjustable parameter in the model, the accuracy of the theoretical curves is surprising. This good agreement is probably due to the accuracy of the SPT expression for the free volume [Eq. (9)].

In Sec. III we concluded that the free volume available to a rod is independent of fugacity. Therefore, the complete phase diagram for the needle-sphere mixtures including the fluid-solid transitions can be obtained using first-order perturbation theory. The coexistence densities for the solidliquid and solid-gas transitions are easily calculated from the chemical potentials and the pressures in Eqs. (8) and (9). The resulting phase diagrams for different rod lengths are shown in Fig. 4. The most obvious result is that a stable liquid-gas two phase region is indeed possible. If the rods get smaller than $0.3 \sigma$ the vapor-liquid phase separation becomes metastable. One can imagine that at this point the rods start to fit in the interstitial spaces of the solid phase. This causes the free energy of the solid-vapor equilibrium to be lower than that of the liquid-vapor equilibrium.

As discussed above the coexistence curves shift to lower fugacities with increasing rod length. The interaction range between the particles becomes larger and the necessary depth of the effective potential to induce phase separation which is governed by the rod fugacity is smaller. The fluid-solid coexistence region becomes wider at high fugacity. This is a common phenomenon in fluid-solid transitions. If the fugacity increases, the free energy in the fluid decreases more than the free energy of the solid. To compensate this, the equilibrium solid phase has to become denser and the fluid phase more dilute. 

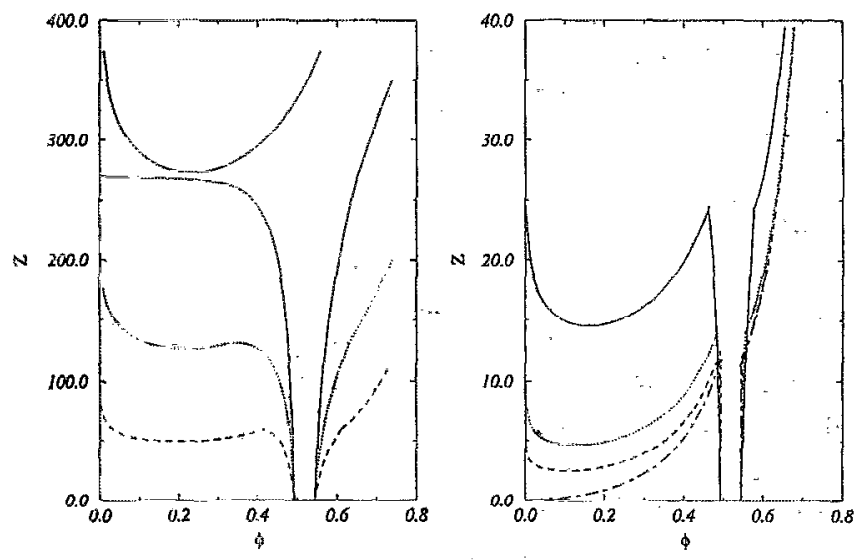

FIG. 4. Phase diagrams of hard-sphere rod mixtures. The fugacity of the rods $z$ is plotted vs the volume fraction of hard spheres, $\phi=\frac{1}{6} \pi \rho_{s} \sigma^{3}$. The lines correspond to the phase boundaries and are obtained by first-order perturbation theory as given in the text. Left picture: solid line $L=0.2$, dotted line $L=0.3$, dashed line $L=0.5$. Right picture: solid line $L=1$, dotted line $L=2$, dashed line $L=3$, and dot dashed line $L \rightarrow \infty$. The solid curve in the left picture is the metastable fluid-fluid binodal for $L / \sigma=1$.

\section{CONCLUSIONS}

When infinitely thin needles are added to a hard-sphere solution a phase separation will occur in a dense, hardsphere-rich, liquidlike phase and a dilute vaporlike phase that contains few hard spheres. This is a pure entropic effect as only hard core interactions are involved. The phase separation is caused by an increase in the available volume for the needles as the spheres cluster. This increase in entropy of the rods will outweigh the loss in entropy of the spheres at high rod fugacity. The fluid-solid transition will preempt this phase separation for a system with a rod length $L / \sigma<0.3$ and a liquid phase will not be stable for these rod lengths. We expect that for very short rods the critical point will reappear in the solid phase. Recent simulations have shown that in systems with very short-range attraction a phase transition can occur between a dense packed crystal and a less dense crystal. ${ }^{16}$ This phase transition is first order, ends in a critical point, and is similar to the liquid--vapor transition.

The features of the phase diagrams of the rodlike polymer are completely comparable with that of the fully flexible chains, ${ }^{4}$ although in the latter case the disappearing of the liquid region is predicted at a polymer radii of gyration $2 R_{g} / \sigma<0.45$. However, the latter estimate is quite crude. It is therefore not obvious that the difference with the value obtained here is significant.

The liquid-gas separation in the rodlike polymer colloid mixture can be described by first-order perturbation theory. This theory predicts the location of the critical point well at rod lengths $L / \sigma<4$ although this accuracy decreases for higher values of $L / \sigma$. The perturbation theory is accurate because the free volume for a rod in a dispersion of spheres is effectively independent of the concentration of rods. Of course this is only valid in the case of ideal polymers. If the polymers would interact with each other the free volume would decrease considerably with the fugacity.
In the limit $L \rightarrow \infty$ the critical point shifts to zero density and zero polymer fugacity. The binodal curve is in this limit equivalent to the equation of state for hard spheres. The fugacity of the rods is equal to the pressure of the coexisting hard-sphere fluid phase because in the vapor phase no spheres are present and the liquid phase is free of needles. This fact may provide an alternative route to obtain the equation of state of hard spheres experimentally by measuring the concentration of rods in the vapor phase of a phase separated mixture of colloids and stiff polymers as a function of the volume fraction of spheres in the liquid phase.

The simulations discussed in this paper show that for mixtures of hard spherical particles and thin needles liquidvapor phase separation will occur. However, one could ob( ject that the limiting case of infinitely thin needles is unrealistic. Hence, it is important to know whether the demixing transition discussed in this paper will still occur if the needles have a finite diameter $D$. In particular, one should consider the possibility that the rodlike particles will undergo a transition from the isotropic to nematic phase. This

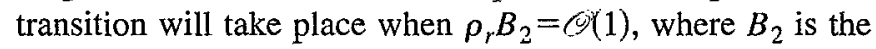
second virial coefficient of the rods. To see at what value of the rod osmotic pressure the isotropic-nematic transition occurs, we consider the Onsager limit of rods with high $L / D$ ratio. ${ }^{17}$ In this limit the osmotic pressure of the rods can be written as

$$
\beta P_{r}=\rho_{r}\left(1+\frac{\pi}{4} D L^{2} \rho_{r}\right),
$$

where we used the diameter of the spheres as a unit of length. The isotropic phase becomes unstable at a density of $(\pi / 4) D L^{2} \rho_{r} \approx 3.3,{ }^{17}$ which corresponds to an osmotic pressure $P_{I N} \approx 18 /\left(D L^{2}\right)$. To observe the liquid gas phase separation, this pressure has to be larger than the critical pressure $P_{c}$ for the phase separation.

In the limit that $D \rightarrow 0$ while $L$ remains finite, the $I-N$ transition occurs at infinite pressure. For rods with a finite diameter, demixing can compete with the $I-N$ transition. To be specific, on the basis of the perturbation theory and Eq. (13) we assume that for $L / \sigma \geqslant 1, P_{c}-2 \sigma e / 3 L$. If we equate $P_{C}$ to $P_{I N}$, we find that demixing is preempted by an $I-N$ transition if $L D / \sigma^{2}>10$. This implies that for small $D / \sigma$ the liquid-gas phase separation occurs well before the $I-N$ transition.

Of course, the finite thickness of the rods will alter the simulated phase diagrams, especially for small $L / D$ ratios. This will probably shift the phase separation region as well as the isotropic-nematic transition to higher fugacities. So we expect that the liquid-gas phase separation still occurs at lower fugacities than the isotropic-nematic transition for reasonable $L / D$ ratios $(L / D>5)$.

\section{ACKNOWLEDGMENTS}

The work of the FOM Institute is part of the research program of FOM and is made possible by financial support from the Netherlands Organization for Scientific Research (NWO). We gratefully acknowledge valuable suggestions by Henk Lekkerkerker. 


\section{APPENDIX: CONFIGURATIONAL BIAS MONTE CARLO IN THE GIBBS ENSEMBLE}

To calculate the phase equilibria directly we use the Gibbs ensemble Monte Carlo simulation approach developed by Panagiotopoulos. ${ }^{8}$ In this method, the coexisting phases are simulated in parallel. $V^{\mathrm{I}}\left(V^{\mathrm{II}}\right)$ denotes the volume of the periodic box containing phase I(II). The total volume is denoted by $V$. The number of spheres and rods in either box is denoted by $N_{s}^{\mathrm{I}}, N_{r}^{\mathrm{I}}, N_{s}^{\mathrm{II}}$, and $N_{r}^{\mathrm{II}}$.

During the simulation ordinary MC displacement steps are performed in both phases and occasionally particles and volume are exchanged between the boxes to maintain, respectively, chemical and mechanical equilibrium. The particle moves inside a box are accepted according to the conventional Metropolis scheme. For trial moves to change the volume, we follow the procedure described in Ref. 9.

The exchange rules are slightly more complicated. The rods can be exchanged without any difficulty and we can use the usual acceptance rule for moving a rod from box I to II:

$$
P_{\mathrm{acc}}=\min \left[1, \frac{V^{\mathrm{\Pi}} N_{r}^{\mathrm{I}}}{V^{\mathrm{I}}\left(N_{r}^{\mathrm{II}}+1\right)} \exp (-\beta \Delta U)\right] .
$$

Ilowever, the spheres cannot be exchanged directly because the insertion probability in both phases will be very low due to the presence of rods. Nearly always a sphere will overlap with one or more needles. To overcome this we can make use of the configurational bias Monte Carlo scheme originally derived by Biben ${ }^{18}$ who in turn based it on a scheme suggested in Ref. 10.

The procedure is as follows. First one tries to move a sphere from one box to the other. When the sphere overlaps with any other sphere the trial move is rejected. Otherwise, rods overlapping with the sphere in its new position are removed and are randomly inserted into the former box in such a way that they would have intersected the sphere in its original position. This procedure creates a bias in the sampling. In the following we show how to correct for this bias. Before discussing particle exchange between two boxes, we focus on a scheme for moving particles within the same box. Suppose we want to swap a sphere with the overlapping needles on the new position. The acceptance rule for this step is governed by detailed balance:

$$
\frac{P_{a \rightarrow b}^{\mathrm{gen}} P_{a \rightarrow b}^{\mathrm{acc}}}{P_{b \rightarrow a}^{\mathrm{gen}} P_{b \rightarrow a}^{\mathrm{acc}}}=\exp \left[-\beta\left(U_{b}-U_{a}\right)\right] .
$$

Here $P_{a \rightarrow b}^{\text {gen }}$ is the probability to generate and $P_{a \rightarrow b}^{\mathrm{acc}}$ the probability to accept the move from configuration $a$ to configuration $b . \Delta U=U_{b}-U_{a}$ is the potential energy difference between $a$ and $b$. To satisfy the detailed balance condition one can use the Metropolis scheme with the acceptance rule

$$
P_{a \rightarrow b}^{\mathrm{acc}}=\min \left[1, \frac{P_{b \rightarrow a}^{\mathrm{gen}} \exp \left(-\beta U_{b}\right)}{P_{a \rightarrow b}^{\mathrm{gen}} \exp \left(-\beta U_{a}\right)}\right] .
$$

In normal simulations $P_{b \rightarrow a}^{\text {gen }}$, the chance of generating configuration $a$ is equal to $P_{a \rightarrow b}^{\text {gen }}$. In the present case the generating probabilities depend on the volume, the number of par- ticles, and the specific configuration. If we try to move $n$ rods starting from configuration $a$, the probability to generate a certain configuration $b$ is given by

$$
P_{a \rightarrow b}^{\text {gen }}=\prod_{i=1}^{n} \frac{\exp \left(-\beta U_{b_{i}}\right)}{Z_{b, i}},
$$

where $U_{b_{i}}$ denotes the energy of the $i$ th moved rod in configuration $b$, the weight factor $Z_{b, i}=\Sigma_{j=1}^{k} \exp \left(-\beta U_{b_{j}}\right)$ plays the role of the single rod partition function. Once we have moved a sphere, we try to move the overlapping rods into the old position of the sphere. For each of the $n$ overlapping rods the weight factor is calculated by inserting rods $k$ times randomly in the overlap volume of the rod and the moved sphere.

Using Eqs. (A2)-(A4) and the relation $\Delta U$ $=\sum_{i=1}^{n}\left(U_{b_{i}}-U_{a_{i}}\right)+U_{b, \mathrm{HS}}-U_{a, \mathrm{HS}}$ the acceptance probability becomes

$$
P_{a \rightarrow b}^{\mathrm{acc}}=\min \left[1, \frac{Z_{b}}{Z_{a}} \exp \left(-\beta \Delta U_{\mathrm{HS}}\right)\right],
$$

where $\Delta U_{\mathrm{HS}}=U_{b, \mathrm{HS}}-U_{a, \mathrm{HS}}$ takes into account the interaction of the moved sphere with all other spheres and $Z_{a}=\prod_{i=1}^{n} Z_{a, i}$ for configuration $a$. For convenience, it is assumed that the number of trial insertions $k$ is the same in both $Z_{a}$ and $Z_{b}$. The weight factors $Z_{a}$ and $Z_{b}$ of the old and the new situation, respectively, must be calculated. Notice that besides the moved sphere the only difference between $a$ and $b$ are the position of the $n$ overlapping rods, so we only need to calculate the weight factors for those rods.

The above scheme is valid for a move within one box. In the Gibbs ensemble scheme the situation is somewhat different. Suppose we want to move a sphere from box II to I. The overlapping $n$ needles in box $I$ in situation $a$ are moved to box II. The probability to generate the new configuration $b$ is now given by

$$
P_{a \rightarrow b}^{\mathrm{gen}}=\frac{1}{N_{s}^{\mathrm{II}}} \frac{1}{V^{\mathrm{I}}} \prod_{i=1}^{n} \frac{\exp \left(-\beta U_{b_{i}}\right)}{Z_{i}},
$$

where the factor $1 / N_{s}^{\mathrm{II}}$ is the probability to pick one of the spheres in box II and the volume factor is due to the random choice of the sphere position in box I. The product in Eq. (A6) gives the probability to move $n$ rods between boxes and is the same as in Eq. (A4). For the reverse move, $P_{b \rightarrow a}^{\text {gen }}$ can be written as

$$
P_{b \rightarrow a}^{\text {gen }}=\frac{1}{N_{s}^{1}+1} \frac{1}{V^{\mathrm{II}}} \prod_{i=1}^{n} \frac{\exp \left(-\beta U_{a_{i}}\right)}{Z_{i}},
$$

where the additional 1 in the first factor arises because in the reverse move one extra sphere can be chosen.

Using $\Delta U=\sum_{i=1}^{n} U_{b_{i}}-U_{a_{i}}+U_{b, \mathrm{HS}}-U_{a, \mathrm{HS}}$ and the definition of the weight factor $Z$, the total probability of accepting a sphere exchange from box II to I is 


$$
P_{a \rightarrow b}^{\mathrm{nac}}=\min \left[1, \frac{V^{\mathrm{I}}}{V^{\mathrm{II}}} \frac{N_{s}^{\mathrm{II}}}{N_{s}^{\mathrm{I}}+1} \frac{Z_{b}}{Z_{a}} \exp \left(-\beta \Delta U_{\mathrm{HS}}\right)\right]
$$

Note that the number of rods does not occur in this acceptance rule, because the rods cannot be chosen freely once a particular sphere has been randomly selected. At first sight it seems strange that the acceptance rule does not contain the number of displaced rods. In the limit in which the rods shrink to ideal point particles, however, it is immediately clear that the thermodynamics of the hard-sphere fluid cannot depend on the configurations of an ideal gas.

The calculation of the $Z_{a}$ and $Z_{b}$ is as follows. The orientation of the rod to be inserted is chosen randomly and it is placed within the overlap volume of the rod and the sphere. This is repeated $k$ times. $Z_{a}$ and $Z_{b}$ are equal to the number of times the rod does not overlap with another sphere in the old configuration $a$ and the new situation $b$, respectively.

To check chemical equilibrium one may want to know the chemical potential of the rods. The chemical potential of the rods is measured by the Widom insertion method which is slightly altered due to the Gibbs ensemble: ${ }^{9}$

$$
\beta \mu=\beta \mu^{0}-\ln \left\langle\frac{V^{\mathrm{I}}}{\left(N_{r}^{\mathrm{I}}+1\right)} \exp (-\beta \Delta U)\right\rangle \text {. }
$$

With the above steps it is possible to calculate phase equilibria in rod-sphere mixtures directly.

${ }^{1}$ S. Asakura and F. Oosawa, J. Chem. Phys. 22, 1255 (1954).

${ }^{2}$ A. Vrij, Pure Appl. Chem. 48, 471 (1976).

${ }^{3}$ H. N. W. Lekkerkerker, W. C-K. Poon, P. N. Pusey, A. Stroobants, and P. B. Warren, Europhys. Lett. 20, 559 (1992).

${ }^{4}$ E. J. Meijer and D. Frenkel, J. Chem. Phys. 100, 6273 (1994).

${ }^{5}$ M. A. Tracy, J. L. Garcia, and R. Pecora, Macromolecules 26, 1862 (1993).

${ }^{6} \mathrm{~T}$. Biben and J. P. Hansen, Phys. Rev. Lett. 66, 2215 (1991).

${ }^{7}$ H. N. W. Lekkerkerker and A. Stroobants, Physica A 195, 387 (1993).

${ }^{8}$ A. Z. Panagiotopolous, Mol. Phys. 61, 813 (1987); A. Z. Panagiotopolous, N. Quirke, M. Stapleton, and D. J. Tildesley, Mol. Phys. 63, 527 (1988).

${ }^{9}$ B. Smit, Ph. de Sinedt, and D. Frenkel, Mol. Phys. 68, 931 (1989).

${ }^{10}$ D. Frenkel, in Computer Simulation in Chemical Physics, edited by M. P. Allen and D. J. Tildesley, NATO ASI Ser. C (Kluwer Academic, Dordrecht, 1993), Vol. 397, p. 93 ff.

${ }^{11}$ B. Widom, J. Chem. Phys. 39, 2808 (1963).

${ }^{12}$ N. F. Carnahan and K. E. Starling, J. Chem. Phys. 51, 635 (1969).

${ }^{13}$ B. J. Alder, W. G. Hoover, and D. A. Young, J. Chem. Phys. 49, 3688 (1968).

${ }^{14}$ D. Frenkel and A. J. C. Ladd, J. Chem. Phys. 81, 3188 (1984).

${ }^{15}$ H. Reiss, H. L. Frisch, and J. L. Lebowitz, J. Chem. Phys. 31, 369 (1959).

${ }^{16}$ P. G. Bolhuis and D. Frenkel, Phys. Rev. Lett. 72, 221 (1994).

${ }^{17}$ L. Onsager, Ann. N.Y. Acad. Sci. 51, 627 (1949).

${ }^{18}$ T. Biben, Ph.D. thesis, Université Claude Bernard-Lyon 1, France (1993). 